\title{
Medicalia Online: tecnicismi medici tra passato e presente*
}

\author{
Isabella Bonati
}

Intellectual cross-pressures genereted by an interdisciplinary outlook liberate a person's thinking from the limiting assumptions of his own professional group, and stimulate fresh vision.

Milgram, Thinking, 103

Interdisciplinarians are forever treating themselves to the intellectual equivalent of exploring exotic lands. [...] To overcome the negative sides of specialization, to retain its vitality, the academy must cultivate interdisciplinary knowledge and research. It must never forget that a vibrant community of scholars - just like a thriving ecosystem - nurtures specialists and generalists, diversity and interconnections.

Nissani, Cheers, 211 e 214

\section{Introduzione}

Con questo contributo si intende offrire una presentazione generale di quella parte del progetto DIGMEDTEXT di cui mi sono maggiormente occupata e a cui sono stata legata nel corso del triennio di ricerca post-dottorale trascorso all'Università di Parma (2013-2016). Focalizzerò quindi l'attenzione sui tratti essenziali del progetto Medicalia Online, nel quale emerge un significativo connubio di testo, contesto e ipertesto dei papiri medici greci, illustrandone l'assetto metodologico, l'impostazione, gli esiti, le prospettive, i punti di forza ${ }^{1}$.

* Questo contributo rientra nel progetto ERC-AdG-2013-DIGMEDTEXT, Grant Agreement No: 339828 (responsabile scientifico Prof.ssa Isabella Andorlini) finanziato dallo European Research Council presso l'Università degli Studi di Parma (vd. la pagina web all'indirizzo http://www.papirologia.unipr.it/CPGM/ERC.html), ma il lavoro di stesura dell'articolo è stato completato durante la mia attuale Post-Doctoral Fellowship presso la North-West University di Potchefstroom, South Africa.

${ }^{1}$ Cf. <http://www.medicaliaonline.unipr.it>. Al progetto Medicalia Online ho dedicato anche i 
Il primo aspetto che occorre mettere in evidenza, ma sul quale si tornerà in seguito (§3), è la stretta, 'viscerale' connessione tra Medicalia Online e le edizioni dei testi medici su papiro contenute nel Corpus dei Papiri Greci di Medicina Online (CPGM), che sta mano a mano confluendo nel Digital Corpus of Literary Papyri (DCLP) ${ }^{2}$. Rispetto al database testuale, i Medicalia si configurano come strumento di supporto e, soprattutto, di approfondimento lessicale, che indirizza verso un contestuale, rinnovato studio filologico e linguistico del vocabolario tecnico, nonché degli stessi contenuti dei papiri medici. Partendo dall'ovvio presupposto che i testi sono fatti di parole, e che la comprensione delle parole veicola la comprensione dei testi, l'acquisizione di una più puntuale definizione delle parole comporta una più puntuale penetrazione dei testi. Da ciò consegue la necessità di creare strumenti appositamente concepiti per studiare le parole in connessione coi testi che le documentano. Nell'àmbito dell'era digitale in cui siamo immersi e nella quale le tecnologie informatiche vengono sempre più frequentemente e proficuamente applicate ai vari settori umanistici, tra cui si annoverano, non ultimi, quelli antichistici, fino a convergere in quel campo di studi e ricerca noto come Digital Humanities (o anche "informatica umanistica" o "umanistica digitale"), le risorse lessicografiche online assumono un indubbio rilievo, eminentemente se collegate a un corpus di edizioni digitali di testi, come è appunto il caso di Medicalia Online.

I papiri medici costituiscono un corpus del tutto peculiare, che comprende porzioni o frammenti più o meno sostanziosi di opere di natura strettamente letteraria, in particolare trattati di autori già noti grazie alla tradizione manoscritta medievale, ma pure di adespoti, come anche, soprattutto, testi tecnici - denominati usualmente 'paraletterari' o, con un senso velatamente (e immotivatamente) dispregiativo, 'sub-letterari' ${ }^{3}$-, quali i manuali, i catechismi e gli scritti legati alla prassi scolastica, le collezioni di ricette redatte in forma di codice di papiro o pergamena, oppure singoli foglietti contenenti prescrizioni che mostrano un carattere 'pratico', nella misura in cui recano indicazioni sui criteri di applicazione ed esplicitano i modi della composizione dei rimedi, o ancora elenchi di prodotti medicamentosi ed etichette di farmaci vergati su pezzetti di papiro, pergamena oppure ostraka. Non meno rilevanti per il tipo di informazioni, più o meno (in)dirette, che ci offrono, spesso assai utili per illustrare e ricostruire la concretezza dell'attività del medico al lavoro, nonché la realtà quotidiana - vera, vissuta, patita - dei pazienti, sono poi i testi propriamente documentari, quali i resoconti e i rapporti stilati dai medici, le petizioni di individui privati, e, soprattutto, la corrispondenza epistolare, che è sovente illuminante in materia di

seguenti contributi: Bonati, Dictionary ed Experience. Cf. inoltre Reggiani, Digital Papyrology I, 129-30 e 275.

${ }^{2}$ Cf. <http://www.litpap.info>. Vd. Reggiani, Digital Papyrology I, 251-3.

${ }^{3}$ Sulla questione si rimanda a Reggiani, Digital Papyrology I, 78. 
comunicazione scritta tra specialisti oppure tra laymen, quando ad essere coinvolte sono questioni riguardanti salute e malattia. Il quadro che si compone è quindi estremamente frastagliato, e riflette una situazione testuale non meno complessa e al pari stimolante. Alla luce di questo, è parso chiaro che la creazione di un dizionario recante succinte definizioni di termini, come all'inizio era stato previsto, non avrebbe soddisfatto le esigenze (né aiutato a delucidare le asperità) esegetiche degli antichi scritti medici. Si è quindi rideterminato lo scopo dei Medicalia, che hanno assunto la forma di una raccolta di studi lessicali con impostazione enciclopedica. Ci si è così concentrati su una selezione di specimina, in modo da fornire una serie di studi esaustivi di una campionatura di vocaboli ${ }^{4}$. L'obiettivo è (stato) quello di produrre non brevi spiegazioni di una molteplicità di parole, come appunto ci si aspetta da un regolare 'dizionario', bensì di elaborare uno 'strumento lessicografico' dall'ampio respiro, mirante a scandagliare nel profondo il lessico tecnico che emerge dai papiri greci (para)letterari e documentari di soggetto medico. I campi semantici a cui ci si è prevalentemente dedicati fino ad ora sono quelli delle patologie, dei contenitori per la preparazione e la conservazione di droghe e rimedi, degli strumenti chirurgici, delle tipologie testuali, delle operazioni chirurgiche e degli aspetti relativi ad esse, ma numerosi altri ne rimangono da esplorare.

Focalizzarsi sulla qualità delle informazioni piuttosto che sulla quantità delle parole è stata quindi una scelta di metodo, dopo aver soppesato potenzialità e limiti di un approccio 'verticale' e qualitativo rispetto a un approccio 'orizzontale' e - indubbiamente (e consapevolmente) - più quantitativo. Si tratta, quindi, di un'operazione di cesello, che richiede l'impegno, la pazienza e l'intervento minuzioso di un orafo piuttosto che il lavoro, pur sempre faticoso, ma assai meno rifinito, di un fabbro; un 'orafo' disposto a rivedere, ritoccare, ricorreggere quanto già fatto puntando al fine di una sempre maggiore 'verticalità' e precisione, ovvero di una sempre più raffinata definizione dei verba: un procedimento, dunque, che mai realmente giunge al termine e coinvolge un prodotto che è costantemente migliorabile e aggiornabile, per cui ogni voce resta, teoricamente, sempre in fieri, ed è spesso l'esito di revisioni,

\footnotetext{
${ }^{4}$ Gli specimina inclusi nel database sono stati selezionati in base alla loro rilevanza sotto molteplici aspetti. Per chiarire le ragioni che hanno guidato il criterio di scelta, si ricordi che alcuni dei vocaboli presentano un ampio numero di attestazioni nelle varie fonti ed hanno uno spettro

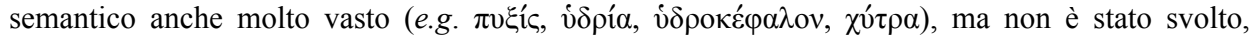
sinora, uno studio sufficientemente inclusivo e comprensivo. Altri termini, invece, sono molto rari

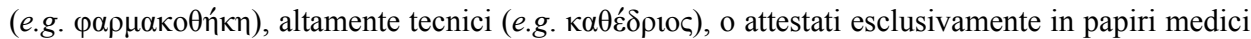

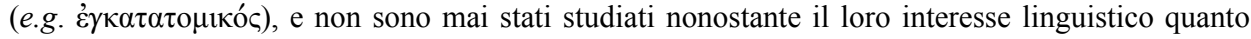
contestuale. Alcuni di questi termini, inoltre, non sono lemmatizzati in alcun dizionario, mentre in altri casi la definizione di essi risulta inesatta rispetto a ciò che un'analisi meticolosa di tutte le fonti e della letteratura secondaria può rivelare. Oltre alla sottoscritta hanno contribuito al database Isabella Andorlini, Anastasia Maravela, Nicola Reggiani e Francesca Bertonazzi.
} 
aggiunte, correzioni e aggiustamenti fatti a più riprese nel tempo. Risulta perciò innegabile il vantaggio offerto dal 'dinamismo' e dalla flessibilità di un database online, che rende sempre aperta la possibilità di intervenire su voci già compilate $^{5}$. Considerando inoltre la vastità del materiale lessicale a disposizione, essendo i papiri un vero e proprio tesoro di dati linguistici restituito dalle sabbie del tempo, la rosa dei vocaboli da analizzare e lo stesso processo lessicografico sono potenzialmente senza fine. Si vuole quindi considerare quanto si è svolto sinora come - auspicabilmente - solo parte di una prima fase di ricerca sul tema, nella speranza che si aprano strade per proseguire questo cammino.

Come è noto e assodato, l'apporto dei papiri alla nostra conoscenza del lessico greco riveste un ruolo notevole, che è stato riconosciuto a partire dal primo riaffiorare di questi documenti nel tardo XIX secolo. Al pari delle altre categorie di papiri, anche quelli medici racchiudono un massiccio potenziale linguistico e si rivelano fonti privilegiate da cui attingere terminologia (a vari gradi) tecnica ${ }^{6}$. Il corpus dei papiri medici greci, infatti, non soltanto ha esteso il nostro sapere riguardo alla letteratura del settore quanto alla pratica medica quotidiana, offrendoci l'opportunità di delineare da un lato un quadro realistico delle patologie che affliggevano gli abitanti della $\chi \omega ́ \rho \alpha$ egiziana, dall'altro di comprendere meglio le terapie farmacologiche e/o i trattamenti chirurgici delle stesse. Sotto il profilo linguistico, esso ha fatto e non mancherà di fare emergere un orizzonte affascinante e talora sorprendente che include nuove attestazioni di parole già conosciute grazie agli scritti pervenutici tramite la tradizione manoscritta medievale, alle quali però magari si aggiungono dettagli e sfumature semantiche inedite, come pure termini non altrove attestati, o almeno assai rari in altri generi di fonti, riportando alla luce elementi del lessico medico greco che si erano perduti. Per questo motivo, un'analisi il più possibile approfondita dei termini technici contribuisce ad affinare la nostra familiarità con il vocabolario medico antico, come, non meno, a favorire l'interpretazione degli stessi testi medici, nonché del contesto che li ha prodotti. Si tratteggia pertanto, come si vedrà meglio in seguito $(\S 3)$, un rapporto di stretta dipendenza 'critica' tra l'esegesi dei testi medici e una puntuale comprensione della terminologia tecnica. Per ribadire un concetto che si è già espresso: una più precisa definizione delle parole favorisce una più precisa comprensione del passo in cui il vocabolo è attestato. Questo potenzia fortemente la nostra consapevolezza filologica dei testi medici e sottolinea la necessità di uno studio 'di cesello' del vocabolario tecnico, nonché l'interesse di un database lessicografico quale risorsa di supporto alle edizioni digitali.

\footnotetext{
${ }^{5}$ Ciononostante, la gran parte dei lemmi già pubblicati online nel database Medicalia verrà anche pubblicata come parte di un volume.

${ }^{6}$ Sul contributo dei papiri medici allo studio della microlingua medica greca, cf. Maravela, Micro-Language.
} 


\section{Metodologia e scopi}

Uno dei punti di forza e di originalità del database Medicalia Online è costituito dall'assetto metodologico che lo caratterizza, nonché dall'elaborazione di un preciso ed efficace metodo d'analisi. Le fondamenta su cui si erge l'architettura di Medicalia Online sono improntate a una marcata interdisciplinarità, la quale si può definire, per riprendere le parole sovente citate di Julie Klein e William H. Newell ${ }^{7}$, come:

A process of answering a question, solving a problem, or addressing a topic that is too broad or complex to be dealt with adequately by a single discipline or profession. [... It] draws on disciplinary perspectives and integrates their insights through construction of a more comprehensive perspective ${ }^{8}$.

L'esigenza di un approccio interdisciplinare in àmbito papirologico è stata messa in luce più volte in anni recenti, e si impone particolarmente necessaria in una disciplina che, per sua natura, è 'tentacolare' e richiede competenze ad ampio raggio, andando ad intersecarsi con molteplici aree di studio del mondo antico (per le quali un approccio interdisciplinare risulta, comunque, altamente proficuo, se non raccomandabile) $)^{9}$. È rilevante infatti ricordare come espressioni quali "broader concept", "broader view", "combination of sources", e, naturalmente, "interdisciplinarity", ricorrano ripetutamente nell'Oxford Handbook of Papyrology curato da Roger Bagnall nel 2009.

Più in generale, il mondo accademico si è spesso interrogato sui vantaggi intellettuali di un approccio interdisciplinare alla conoscenza e alla ricerca, per evitare l'eccessiva frammentazione - col rischio di 'sclerotizzazione' - delle discipline e delle scienze quando confinate entro perimetri troppo rigidamente specialistici. Sono significative, per esempio, le osservazioni - in questo caso rivolte alla biologia e alle scienze politiche, ma non meno applicabili ad altre branche del sapere - di Kenneth P. Ruscio:

Specialization is both a blessing and a course for the academic profession, but lately its disadvantages have received the most attention. [...] Some links facilitate integration and thereby prevent specialization from becoming narrow-mindedness. [...] We need to reconceptualise our model of disciplinary growth and specialization, adopting a more organic model that

\footnotetext{
${ }^{7}$ Per approfondire l'argomento si rimanda in particolare a Newell, Theory, 1-25 e State, 22-43; Klein, Interdisciplinarity e Mapping; Hübenthal, Thought, 55-75; Repko, Research.

${ }^{8}$ Klein/Newell, Studies, 393-4.

${ }^{9}$ Sull'urgenza di uno studio interdisciplinare nei diversi settori antichistichi, cf. per esempio Gentili, Poesia, 329-45 e Pagnotta, Lezione, 850-2 con bibliografia.
} 
accounts for the intricate links among many specializations. Our current mechanistic model divides disciplines into numerous blocks of specializations; it is inaccurate [...] and misleading ${ }^{10}$.

È istruttivo anche richiamare alcuni passi di un articolo di Moti Nissani che si pone come una "self-contained, comprehensive defense of interdisciplinary knowledge and research", sottolineando, in una sorta di decalogo, le varie ragioni dell'importanza di questo tipo di approccio:

1. Creativity often requires interdisciplinary knowledge. 2. Immigrants often make important contributions to their new field. 3. Disciplinarians often commit errors which can be best detected by people familiar with two or more disciplines. 4. Some worthwhile topics of research fall in the interstices among the traditional disciplines. 5. Many intellectual, social, and practical problems require interdisciplinary approaches. 6 . Interdisciplinary knowledge and research serve to remind us of the unity-of-knowledge ideal. 7. Interdisciplinarians enjoy greater flexibility in their research. 8. More so than narrow disciplinarians, interdisciplinarians often treat themselves to the intellectual equivalent of traveling in new lands. 9. Interdisciplinarians may help breach communication gaps in the modern academy, thereby helping to mobilize its enormous intellectual resources in the cause of greater social rationality and justice. 10. By bridging fragmented disciplines, interdisciplinarians might play a role in the defense of academic freedom. The case against interdisciplinary knowledge and research is made up of many intrinsic drawbacks and practical barriers. Taken together, these rewards, drawbacks, and barriers suggest a mild shift in the contemporary world of learning towards interdisciplinary knowledge and research ${ }^{11}$.

Ciò, inoltre, può aiutare a comprendere meglio comprendere le problematiche dell'oggetto indagato, nonché incoraggiare a non respingere $a$ priori un ideale di unità del sapere, il quale, pur essendo un traguardo di per sé utopistico in epoca moderna, almeno suggerisce la possibilità di approcciarsi allo scibile con l'intenzione o il desiderio di abbracciare un panorama intellettuale quanto più possibile ampio e comprensivo, senza restringere lo sguardo ai soli dettagli questo è il rischio di una specializzazione severamente 'esclusiva' -, ma inglobando quei dettagli in seno al tutto che li completa e li circonda, optando quindi per un'ottica 'inclusiva' ${ }^{12}$ :

\footnotetext{
${ }^{10}$ Ruscio, Specializations, 29 e 43-4.

${ }^{11}$ Nissani, Cheers, 201.

${ }^{12}$ Nissani, Cheers, 209-10, da cui sono tratti i brani riportati di seguito.
} 
Many complex or practical problems can only be understood by pulling together insights and methodologies from a variety of disciplines. Those who forget this simple truth run the intellectual risk of tunnel vision.

It is of course impossible, in our age, to become an expert in everything. But [...] if we forget how much we don't know; if we forget how much we cannot know; if we don't set for ourselves, in principle at least, the ideal of the unity of knowledge; we lose something of great importance. By persistently aiming at the hazy target of omniscience, interdisciplinarians help us remember these things. They thus spur us to see the various components of human knowledge for what they are: pieces in a panoramic jigsaw puzzle.

Ne consegue che una tale metodologia, così atta a integrare i migliori insights delle varie disipline per produrre un ritratto estensivo, policromo, multisfaccettato dell'oggetto di studio, incentivi ad abbattere barriere, piuttosto che a erigerle, e a costruire ponti tra i vari campi della conoscenza. È questo appunto il caso dell'approccio che innerva Medicalia Online. Infatti, sebbene gli studi lessicali prendano avvio dal terreno papirologico, un criterio sistematicamente e profondamente interdisciplinare ne ispira l'intrinseca natura. Ciò contribuisce a espandere l'orizzonte del database lessicografico a un vasto ventaglio di prospettive (papirologica, linguistica, archeologica, storico-scientifica), che sono l'esito di un'analisi critica e di un esame comparativo e 'dialogante' del complesso delle evidenze disponibili, da quelle scritte (papiri, passi letterari soprattutto di autori medici, iscrizioni e tituli picti) ai reperti archeologici che si fanno testimoni di una determinata pratica medica relativa al termine oggetto di studio. Una metodologia che, quindi, potrebbe essere definita 'sinergica', dal momento che mira a far convergere sullo studio di ogni verbum l'utilizzo di ogni testimonianza reperibile, così concorrendo a fare luce sul contesto complesso e multiculturale della medicina greco-romana in Egitto. Ciò deriva, appunto, da un forte senso di 'dialogo' e 'cooperazione' tra varie discipline, ovvero di 'integrazione', che è uno degli elementi che meglio caratterizza lo spirito dell'interdisciplinarità. Diversi sono infatti i settori antichistici coinvolti oltre alla papirologia: letteratura e filologia classica, linguistica, epigrafia, archeologia e cultura materiale, medicina e storia della scienza. Di riflesso, da un lato l'orizzonte della potenziale readership risulta ampliato, includendo studiosi o appassionati dei rispettivi settori, dall'altro questo sguardo 'globale' sulle testimonianze del passato solleva quell'urgenza di una concezione unitaria e integrata delle scienze antichistiche cui prima si è accennato.

Un'altra parola chiave che connota Medicalia Online, e che è già emersa in queste pagine, è 'verticalità'. Questo 'dialogo' criticamente comparativo e 'verticale' indirizza verso una visione quanto più ampia di ogni lemma e incrementa la nostra comprensione degli aspetti tanto testuali quanto concreti del 
mondo antico. Una tale operazione, che potrebbe essere descritta come una 'archeologia delle parole', si traduce inoltre nello sforzo di 'rivitalizzare' il passato, rendendolo meno 'polveroso' e irrimedabilmente distaccato da noi, nonché, quindi, più accessibile al presente. Questo significa - repetita iuvant - il tentativo (non sempre, bensì sovente, soddisfacente e produttivo) di cogliere e di 'ricostruire' l'antichità come un insieme di verba e realia. Possono, pertanto, le testimonianze del passato tornare a 'parlare', recuperando quella voce che il tempo, lo scorrere di secoli o millenni, sembrava aver disperso, e invece ha solo imprigionato e ovattato nella sabbia, e la cui eco è ancora - se non 'viva' almeno 'vivida' per chi è disposto ad ascoltare ${ }^{13}$.

Inoltre, prendendo avvio dall'evidenza dei papiri e da una scrupolosa analisi linguistica, particolare attenzione è riservata all'evoluzione e alla sopravvivenza dei termini analizzati nelle lingue moderne e nel lessico scientifico contemporaneo. Uno degli scopi principali di Medicalia Online consiste infatti nel gettare nuova luce sul passato ed il presente dei tecnicismi medici seguendone gli sviluppi lungo i secoli. Tale confronto in senso diacronico, dall'antichità ai giorni nostri, può portare risultati non trascurabili anche dal punto di vista della storia della medicina, come per esempio consentendo un raffronto accurato tra la concezione antica e quella moderna di una patologia. Uno specimen eloquente è

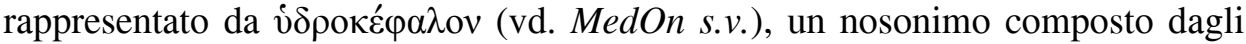
elementi $0 \delta \omega \rho$, "acqua", e $\kappa \varepsilon \varphi \alpha \lambda \eta$, "testa", che esprimono rispettivamente la causa e la localizzazione della patologia, ovvero - letteralmente - "acqua nella testa". Il termine 'idrocefalo' è perdurato sino a noi, a partire dalle prime attestazioni nella letteratura medica antica, adattandosi foneticamente alle varie lingue di arrivo, a cui è pervenuto o direttamente dal greco o tramite il tardo latino.

La sola evidenza papiracea sull'idrocefalo è rappresentata da GMP I 6 (M-P ${ }^{3}$ 2340.01, LDAB 10278, TM 69007), un frammento di catechismo medico databile al tardo II - inizio III secolo d.C., in cui questa condizione è discussa ai rr. 3-18 e il termine è esplicitamente menzionato ai rr. 5 e $7^{14}$. Per meglio comprendere la traiettoria semantica che il vocabolo ha percorso in senso diacronico, può essere utile tracciare una breve panoramica delle più antiche testimonianze del termine, con qualche annotazione linguistica.

La prima descrizione scientifica dell'idrocefalo - malattia comune nell'antichità, come è confermato, oltreché dalla frequenza con cui essa è descritta dalle fonti, dai numerosi ritrovamenti di teschi idrocefali databili al periodo tra il 2500 a.C. e il 500 d.C. - si trova nel Corpus Hippocraticum (Morb. II $15=$ X 2 149,1-

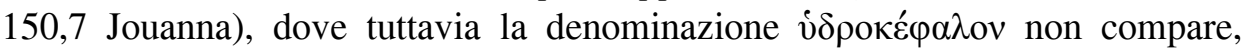
benché l'autore si riferisca chiaramente a questa condizione con la perifrasi

\footnotetext{
${ }^{13}$ Sul tema della rivitalizzazione del passato, cf. Bonati, Parola.

${ }^{14} \mathrm{Su}$ questo papiro e le problematiche ad esso connesse, cf. l'ed.pr. di Hanson/Mattern, Catechism, 71-83.
} 


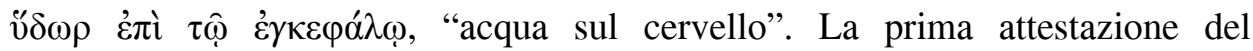
composto si trova invece in un passo della sezione medica dei Problemata pseudo-aristotelici (cf. Ps.Aristot. Probl. I 16,6-11 = 861a,14-19 Bekker) nella

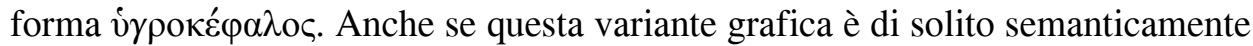

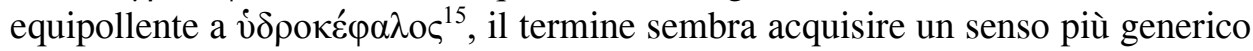
nel luogo aristotelico. Non pare infatti riferirsi alla condizione idrocefalica, ma al fatto che i bambini hanno, di norma, la testa umida, sicché il composto sembrerebbe perdere il consueto valore di terminus technicus per assumere un senso letterale ${ }^{16}$. Le più antiche occorrenze del nosonimo nell'usuale forma

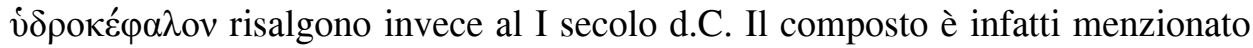

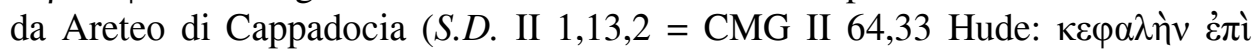

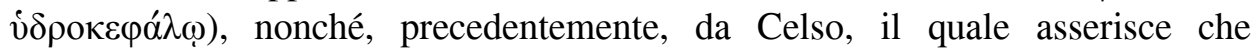

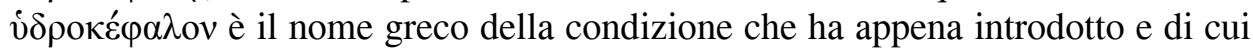
si accinge a fornire una diligente descrizione, comprensiva di eziologia e sintomatologia (cf. Cels. Med. IV 2,4-9 = CML I 152,16-153,27 Marx).

Scandagliando le fonti si può inoltre osservare un - apparentemente inatteso -

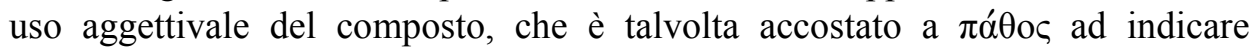

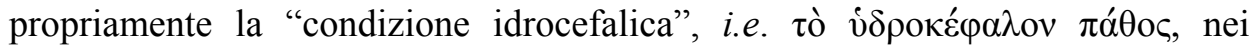
compilatori tardo-antichi, da Oribasio (V d.C.) a Paolo d'Egina (VII d.C.) ${ }^{17}$.

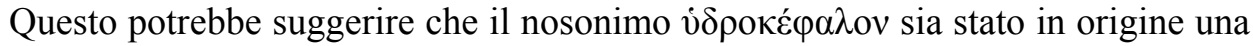
forma ellittica di questa perifrasi, facendo quindi supporre che l'utilizzo aggettivale del termine per designare la patologia, sebbene non attestato nelle fonti superstiti prima di Oribasio, sia in realtà più antico, e che il sostantivo

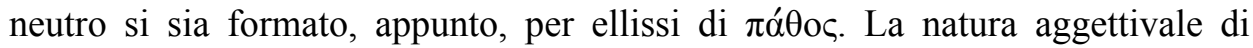

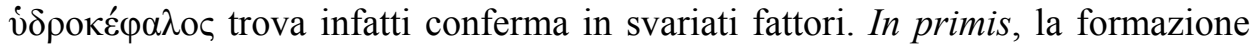

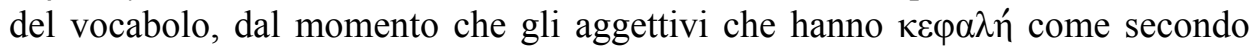
membro terminano di regola in $-\kappa \varepsilon ́ \varphi \alpha \lambda o \varsigma^{18}$. Inoltre, sotto un profilo sintattico,

${ }^{15}$ Per citare qualche esempio, la variante $v \gamma \rho$ - si trova - ed è accettata dai moderni editori - in Sor. Gyn. IV 3,1,4 e 11,3,4 (CMG IV 132,12 e 142,13 Ilberg), ma il secondo passo è emendato in

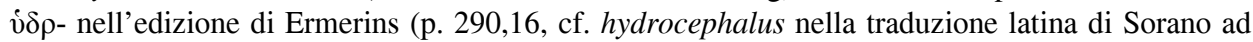
opera di Musc. XVII 29,10 = 93,5 Rose) e in Alex.Trall. X (II 453,7 Puschmann).

${ }^{16} \mathrm{D}$ 'altro lato, il contesto stesso e il tema trattato - il fatto che la ftiriasi sia copiosa nella testa, perché è la regione più umida - supportano questa impressione, come pure alcune asserzioni

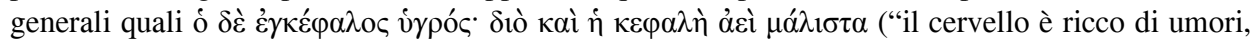

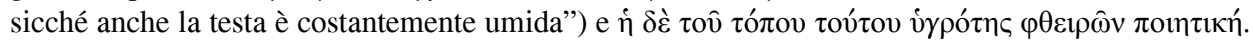

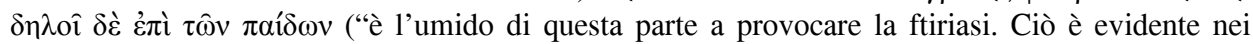
bambini"), e non meno l'antica concezione secondo cui la causa della ftiriasi era appunto l'abbondante umidità del corpo, fatto che si riferisce ad una condizione generale e non certo al caso specifico dell'idrocefalo.

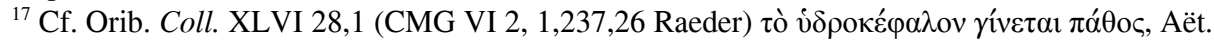

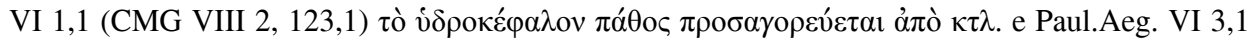

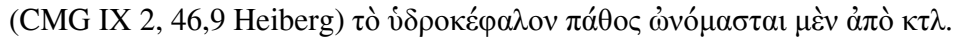

${ }^{18}$ Diversi dei quali sono attestati negli autori medici, quali, e.g., $\alpha \delta \rho \circ \kappa \varepsilon ́ \varphi \alpha \lambda o \varsigma, \alpha \dot{\alpha} \kappa \varepsilon \varphi \alpha \lambda o \varsigma$, 


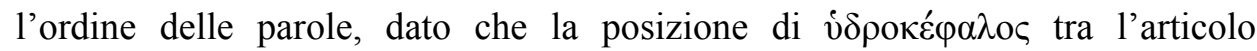
determinativo e il sostantivo lo rende un adjectival modifier di $\pi \alpha ́ \theta$ oc. Infine, il parallelismo con accostamenti in cui il composto ha certamente funzione di noun-modifier ${ }^{19}$.

Nonostante la continuità lessicale di questo nosonimo, l'analisi attenta delle fonti greche e latine, supportata dallo studio della letteratura secondaria, ha evidenziato e confermato quanto l'antica nozione di 'idrocefalo' fosse differente da quella della medicina moderna, e includesse un range di malattie assai più vasto rispetto all'attuale, come tumori extracranici ed ematomi subcutanei, che non sono attualmente classificati come idrocefalo. Pertanto, il significato antico

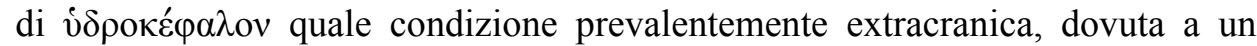
accumulo di fluido extracerebrale, come emerge per esempio nella descrizione di Celso già menzionata, non si sovrappone con l'idrocefalo della terminologia medica moderna. Qui il medicismo designa invece una patologia che è contraddistinta da un eccessivo accumulo di liquor cefalorachidiano in aree endocraniche, ovvero nello spazio sub-aracnoideo (idrocefalo comunicante) e/o nelle cavità ventricolari (idrocefalo non comunicante), che così si dilatano causando ipertensione intracranica e danni al tessuto cerebrale.

Un ulteriore scopo dei Medicalia Online è quello di indagare le varie strategie linguistiche adoperate dagli antichi per la creazione e lo sviluppo della terminologia tecnica della microlingua medica. Alcuni degli specimina esaminati sono infatti risultati assai utili per illustrare queste strategie, come, segnatamente, il conio di neoformazioni tramite composizione (e.g. il succitato

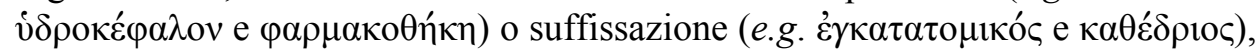
oppure il prestito di termini già esistenti da altri campi semantici, ovvero l'utilizzo di metafore (e.g. $\pi \tau \varepsilon \rho u ́ \gamma ı v$ e $\tau \varepsilon \rho \eta \delta \omega ́ v)^{20}$.

Ancora un altro aspetto riguarda l'osservazione della relazione - mirante a individuare punti di divergenza e di contatto - tra la terminologia medica documentata dai papiri e il linguaggio tecnico - sovente più 'sofisticato' adoperato dagli scrittori medici che ci sono stati consegnti dalla tradizione manoscritta medievale, dal Corpus ippocratico alle opere dei compilatori della tardo-antichità, i quali, prima del riaffiorare dei papiri medici dalle sabbie dell'Egitto, hanno costituito l'unica fonte per conoscere e ricostruire il vocabolario medico greco. Alla luce di questo, quindi, gli studi lessicali dei Medicalia Online ci permettono di esplorare l'affascinante e proficuo contributo dei papiri medici alla nostra conoscenza della microlingua medica antica.

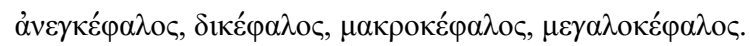

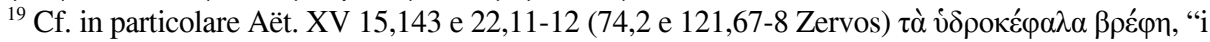

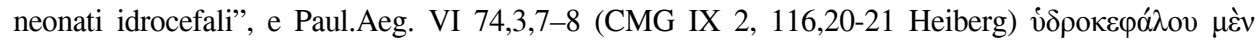

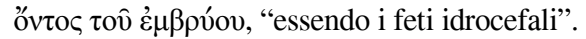

${ }^{20} \mathrm{Su}$ questi temi, cf. rispettivamente Bonati, Composti e Metafora, 84-100.
} 
Volendo ora tratteggiare alcune delle difficoltà che un approccio così dettagliato e interdisciplinare comporta, si richiami per esempio il fatto che questa 'verticalità' metodologica richieda un'ovvia - se non connaturata - necessità di vagliare il complesso delle occorrenze in modo da comprendere quali siano effettivamente utili, nonché in grado di fornire apporti originali, e quali invece meno, rendendo conseguentemente più arduo il compito di riuscire a gestire e maneggiare in maniera funzionale e appropriata un così vasto novero di fonti, specialmente nel caso di termini estremamente attestati. Un altro fattore di non minore rilievo è rappresentato dall'urgenza, che non raramente si impone, di creare affinità con, ed anzi accedere a settori di studio e specializzazione che sono di per sé estranei alla formazione accademica di classicisti e papirologi. Ciò implica il bisogno di avvalersi della consulenza, se non collaborazione, di specialisti versati nei vari campi medici coinvolti per ciascuno studio lessicale. Un aspetto talora non semplice, ma comunque stimolante, che è insito nella natura stessa di una ricerca interdisciplinare, come ricordano ancora le parole di Nissani:

In some cases, interdisciplinary research requires cooperation of experts with different disciplinary backgrounds and different ways of thinking - a notoriously difficult undertaking. Interdisciplinary knowledge and research are demanding. To keep reasonably abreast of just two fields, for instance, requires tremendous investment of time and intellectual energy ${ }^{21}$.

È particolarmente calzante e rappresentativo il caso in cui, studiando un nosonimo, risulta problematico comprendere e determinare esattamente la patologia che si celava dietro quel nome, nonché soprattutto individuare a cosa possa corrispondere quell'antica malattia nella medicina moderna. Un esempio emblematico è costituito da $\tau \varepsilon \rho \eta \delta \omega ́ v v^{22}$.

La sola testimonianza papiracea sulla $\tau \varepsilon \rho \eta \delta \dot{\omega} v$ è lo stesso frammento di catechismo cui già si è fatto cenno, GMP I 6. Appena conclusa la discussione

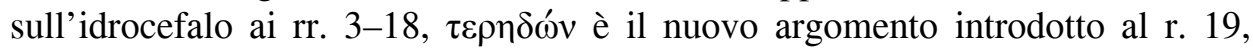
verosimilmente in forma di domanda $\tau$ í $\dot{\delta} \sigma \tau \dot{\eta} \dot{\eta} \tau \rho \eta \delta \omega ́ v$; ("che cosa è la teredon?"), ma il papiro si interrompe appena dopo la seconda menzione del vocabolo, al r. 20, che coincide con l'inizio della definizione.

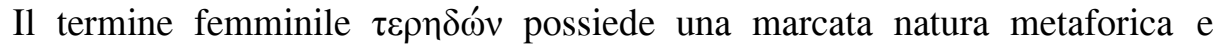
deriva dalla denominazione greca del verme del legno. Questo sostantivo, da cui si formano tre derivati prettamente tecnici, che sono attestati esclusivamente negli autori medici a partire dal I sec. d.C. ${ }^{23}$, è etimologicamente connesso

\footnotetext{
${ }^{21}$ Nissani, Cheers, 212-3.

${ }^{22}$ Cf. MedOn s.v.

${ }^{23}$ Ovvero il verbo $\tau \varepsilon \rho \eta \delta o v i ́ \zeta o \mu \alpha 1$, che significa "essere mangiato dai vermi" quando si riferisce ad alberi e piante (cf. Dsc. M.M. I 1,1,13, nonché I 127,3,4 e IV 153,2,6 = I 6,9, nonché 127,6 e II
} 
all'idea del "perforare" e deriva dalla radice disillabica indoeuropea *terh("forare") $)^{24}$, su cui si innesta il suffisso espressivo $-\eta \delta \omega ́ v$, che è abbastanza comune in nomi di insetti ${ }^{25}$.

Il vocabolo, in senso nosologico, designa la cosiddetta "carie delle ossa", una condizione medica che, stando alle fonti, sembra sia stata abbastanza comune nell'antichità, come pure parrebbe confermato dall'analisi paleopatologica di resti umani dal Neolitico in avanti. Sono diverse le ipotesi sull'origine metaforica di questo nosonimo, come un'analogia morfologica tra l'apparenza del legno mangiato dal verme e l'osso cariato; il paragone per così dire "funzionale" tra il verme stesso che divora il legno e la malattia che assale il corpo; oppure il temine potrebbe anche celare la traccia di un'arcaica teoria eziologica sulla genesi della malattia, come per esempio la credenza che fosse effettivamente un verme a ridurre le ossa in quello stato.

Nel tentativo di rintracciare un parallelismo tra $\mathrm{i}$ dati forniti dalle fonti

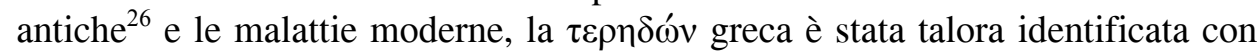
un tipo di osteomielite che affligge in particolare il cranio, come si evince, per esempio, da quanto afferma Mirko D. Grmek:

L'importance que les texts hippocratiques accordant à la carie de la tête nous fait croire que chez les Grecs le siege de predilection de l'ostéomyélite était le crâne. La raison en est probablement la fréquence très élevée des infections de la region stomato-rhino-otologique, notamment chez les enfants ${ }^{27}$.

Ovviamente, come già si anticipava, non è facile comprendere se - e fino a che punto - questa identificazione sia appropriata, data la duplice difficoltà da un

299,1 Wellmann; Orib. Coll. XI a 14,12 = CMG VI 1,2, 107,30 Raeder; Paul.Aeg. VII 4,7,4 = CMG IX 2, 277,27 Heiberg), ed "essere cariato", quando è riferito alle ossa (cf. e.g. Orib. Coll. XLIV 20,10,4, nonché 20,22,2, 20,64,2 e 64,4-65,1 = CMG VI 2,1, 135,30, nonché 136,36, 140,30

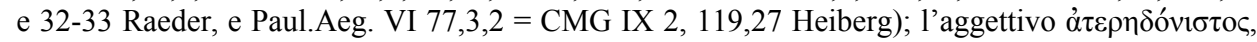
"non divorato dai vermi", che, pur essendo esclusivamente attestato in autori medici (cf. Dsc. M.M. I 16,1,6, nonché II 160,1,6 e III 2,1,4 = I 22,5, nonché 226,10 e II 4,2 Wellmann; Orib. Coll. XI $\zeta 2,5$, nonché $\kappa 31,5$ e XII $\rho 1,1=$ CMG VI 1,2, 103,34, nonché 112,20 e 136,2 Raeder, e Id. Syn. II 56,24,1 = CMG VI 3, 45,17 Raeder; Aët. II 196,46 = CMG VIII 1, 224,21 Olivieri), nei quali è sempre applicato alle radici e al legno di piante e alberi, non appartiene al lessico medico

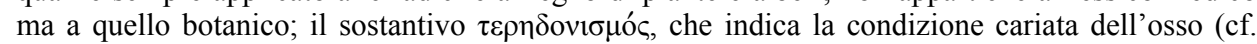
Orib. Coll. XLVI 22 = CMG VI 2,1, 232,18 Raeder).

${ }^{24}$ Questa radice si ritrova anche in altre parole greche, quali, per esempio, i verbi $\tau \varepsilon i ́ p \omega$

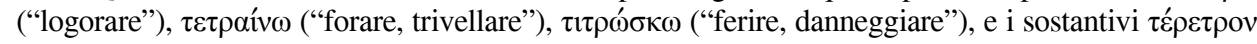
("trapano, trivella") e $\tau \rho \hat{\mu} \mu \alpha$ ("foro, apertura"), vd. inoltre il latino tero e il sanscrito trnnatti.

${ }^{25}$ Cf. e.g. $\dot{\alpha} v \theta \eta \delta \omega ́ v$ ("ape"), $\alpha \dot{\alpha} \theta \rho \eta \delta \omega ́ v$ ("calabrone"), $\pi \varepsilon \mu \varphi \rho \eta \delta \omega ́ v$ ("vespa”).

${ }^{26}$ Le prime descrizioni scientifiche e le prime attestazioni della $\tau \varepsilon \rho \eta \delta \omega ́ v$ risalgono al Corpus Hippocraticum, cf. Morb. II 7 e 24 (X.2 138,9-15 e 188,1-9 Jouanna), nonché Epid. VII 35 (V 402,17-404,13 L.), dove tuttavia il termine non è esplicitamente menzionato.

${ }^{27}$ Grmek, Maladies, 193. 
lato di determinare esattamente la malattia che si celava dietro il suo nome antico e, dall'altro, di identificare a che cosa esso possa corrispondere nel lessico moderno. Bisogna quindi usare cautela, senza la pretesa di pervenire a una risposta definitiva. Resta il fatto che, come mi ha confermato la consulenza di uno specialista in ematologia, diversi aspetti e sintomi della "carie delle ossa"

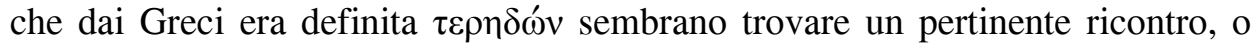
almeno non contraddire, svariati dei sintomi della moderna osteomielite, per cui l'ipotesi sembrerebbe percorribile.

\section{La piattaforma online e il layout delle schede}

Il database è costruito sulla piattaforma open-access TemaTres e offre diverse opzioni di navigazione. L'homepage presenta una suddivisione in tre macrocategorie, ognuna delle quali è ulteriormente ripartita in sottocategorie, e ciò si traduce in una sorta di classificazione tassonomica dei termini: "Lexicalia" (e.g. containers, ingredients, instruments, termini technici), "Medical branches" (e.g. gynaecology, ophthalmology, pathology, pharmacology, surgery), "Text typologies" (e.g. adespota, catechism, documentary texts, prescription). Un singolo termine può inoltre essere subordinato a due o più sottocategorie, ed è così ricercabile in ciascuna di esse. Il secondo modo per navigare nel database segue il criterio alfabetico. Cliccando sulla lettera greca o latina a cui si è interessati, nella parte superiore o inferiore, a sinistra, dell'homepage, si ottiene la lista dei vocaboli che iniziano con quella lettera, siano essi greci, latini o inglesi. Infine, è possibile anche usufruire delle opzioni full-text search e "Advanced search", che si trovano all'estremità superiore della pagina. Quest'ultima consiste in un menu a discesa cliccando sul quale si ottiene una 'tendina' di elementi che consentono di affinare e circoscrivere i parametri di ricerca: selezionando "Term" si restringe la ricerca alle voci, mentre "Meta-term" permette di limitarla alle categorie, "Non preferred term" alle voci secondarie connesse a quella principale, quali varianti e diminutivi, e "Note", infine, alle sezioni tematiche del lemma.

La struttura lessicografica dei lemmi è innovativa e riflette l'approccio metodologico interdisciplinare che ispira la natura di Medicalia Online. La tipologia di layout che è stata codificata è pertanto uno dei fattori di originalità del database, e ad essa si è pervenuti a seguito di numerosi tentativi. Questi rientrano nella 'preistoria' di Medicalia Online, che precede l'avvio effettivo del progetto DIGMEDTEXT all'Università di Parma. I primi passi furono infatti mossi tra l'agosto 2012 e l'aprile 2013 in terra norvegese, durante un soggiorno di ricerca presso l'Università di Oslo finanziato da un "Yggdrasil grant" del Norwegian Research Council. In quel periodo ho avuto il piacere di lavorare sotto la supervisione della Prof.ssa Anastasia Maravela, la quale, con fruttuosi confronti e consigli, ha fortemente contribuito alla messa a punto del definitivo layout. Questo è quindi stato raggiunto mediante ripetuti aggiustamenti, a cui 
hanno concorso anche i feedback ottenuti in sede di discussioni e presentazioni a workshop internazionali ${ }^{28}$, per cui è stato possibile organizzare i contenuti delle schede nel modo più efficace da un punto di vista della readership. In quella prima fase mi sono occupata di alcuni dei contenitori d'uso medico che compaiono nel database ${ }^{29}$. Queste prime schede, poi revisionate e aggiornate nel tempo, sono servite da modello per testare vantaggi e svantaggi, utilità e usabilità del database stesso.

Ogni lemma fornisce un'ampia panoramica delle parole esaminate ed è essenzialmente comprensivo. Questo tipo di layout è stato inoltre pensato per essere il più possibile user-friendly. Un buon livello di user-friendliness è infatti un prerequisito essenziale quando uno strumento lessicografico si presenta complesso e raccoglie una mole di informazioni così cospicua come i Medicalia. In aggiunta, questa articolazione dei lemmi risulta applicabile alle varie categorie lessicali e ai diversi campi semantici. Tale applicabilità mescola dunque elementi di rigidità, ovvero la codificazione di uno standard - l'impostazione dei lemmi secondo un'organizzazione e una suddivisione che si replica ogni volta -, e di elasticità: il fatto che con minime varianti formali la stessa struttura possa essere adeguata alle varie categorie lessicali senza uscirne snaturata. Ogni voce consiste delle sezioni tematiche ("Notes") riportate di seguito:

- "Variants" include una lista di varianti, siano esse grammaticali, come i diminutivi, o fonetiche, quali assai spesso si riscontrano nei papiri, nonché traslitterazione o forme latine del vocabolo, e corradicali di pertinenza medica, qualora presenti;

- "General definition" fornisce una definizione sintetica, sul modello di quelle di un dizionario stricto sensu, che consente al fruitore di venire a conoscenza dei contenuti principali concernenti il termine, come il suo significato 'immediato', prima che egli si addentri, nel caso desideri farlo, nella lettura del lemma;

- "Language between text and context" è la sezione più marcatamente linguistica $\mathrm{e}$ contiene discussioni e osservazioni sull'etimologia, la morfologia, la semantica, le varianti, i derivati e i corradicali del termine, la storia linguistica di esso fino all'epoca moderna, nonché gli sviluppi diacronici dei suoi valori semantici;

- "Testimonia - a selection of representative sources" contiene una lista di passi greci e latini accompagnati da traduzione inglese, e attinti ad ogni tipo di

${ }^{28}$ Le prime presentazioni ufficiali del progetto Medicalia Online sono state: "Trying out layouts for the Medicalia Online lemmata: the example of pharmakotheke", esposta al kick-off workshop "Strengthening Research Capacity in the Papyrus Collection of the Oslo University Library" (3-5 dicembre 2012), presso l'Università di Oslo, e "The vocabulary of some representative ancient Greek containers for food, salsamenta, aromata and medicines", presentata a un workshop organizzato dal Dipartimento di Archeologia (Institutt for arkeologi, konservering og historie - IAKH) della medesima Università (9 febbraio 2013).

${ }^{29}$ Sul tema cf. Bonati, Lessico. 
fonte che attesti il termine, si tratti di testi letterari, papiracei o di iscrizioni. Il criterio di selezione è guidato dalla rilevanza medica del passo e dal grado di funzionalità ai fini del discorso;

- "Commentary" è la sezione più estesa del lemma e mira a contestualizzare il vocabolo all'interno delle fonti, per meglio definirlo, nonché, attraverso queste ultime, a sviscerarne gli apetti intrinseci e, possibilmente, sinora sottovalutati o non appropriatamente messi in luce, proiettandolo nel più ampio panorama storico-scientifico e cogliendone gli elementi peculiari in relazione al tessuto testuale. Per raggiungere questo scopo, la sezione si articola in due sottosezioni. La prima ("[the term] and its medical sources") si concentra su quanto e che cosa le antiche evidenze sanno far emergere a proposito del vocabolo in analisi, concentrandosi anche sull'indagine degli eventuali mutamenti semantici avvenuti nel corso dei secoli, dalle più antiche attestazioni alla tarda antichità, e sui confronti tra il/i significato/i antico/hi e moderno/i di esso, qualora il vocabolo, con i necessari adattamenti fonetici, sia sopravvissuto nelle lingue moderne, eminentemente nel solco delle varie branche specialistiche della medicina, o comunque in settori - e quindi linguaggi - tecnici. La seconda sottosezione ("[the term] in practice") è rivolta al lato più 'pratico' del termine, ovvero è tesa a delineare la connessione tra il verbum esaminato e la sua dimensione concreta. Per accennare solo a qualche specimen evidente, questa operazione implica la ricostruzione materiale del relativo oggetto in caso di parole che denotino, per esempio, res medicae, quali contenitori adoperati per la preparazione o la conservazione di rimedi o sostanze terapeutiche, oppure strumenti chirurgici, ma anche i metodi di trattamento e le procedure chirurgiche in caso di vocaboli indicanti disordini o patologie, prestando particolare attenzione a paralleli, divergenze e innovazioni occorsi lungo la storia della medicina;

- "Bibliography" si articola in "Lexicon entries", i.e. dizionari, glossari, opere etimologiche etc., e "Secondary literature", i.e. studi più esaustivi e specifici inerenti quel termine o quell' argomento;

- "CPGM/DDbDP reference(s)" include la lista delle evidenze papirologiche di àmbito medico contenenti la parola. Dal momento che alcuni di questi termini ricorrono solo nel CPGM Online, mentre altri appaiono anche o esclusivamente in testi documentari che coinvolgono tematiche mediche, che sono dunque contenuti nel DDbDP, si potrà - auspicabilmente - linkare le "DDbDP references" al corrispondente testo che si trova in Papyri.info, e le sigle delle evidenze letterarie o paraletterarie ("CPGM references") ai testi che stanno confluendo nel DCLP, in cui, allo stesso modo, saranno e, in parte, già sono inseriti i link che rimandano alle schede di Medicalia Online. Per quanto concerne quest'ultimo caso, al momento attuale è solo possibile creare link tra i lemmi e le relative parole linkabili, contraddistinte da sottolineatura, all'interno del front matter delle edizioni digitali in DCLP, come, parimenti, tra queste e i lemmi in Medicalia Online. Ci si augura tuttavia che in un futuro non troppo prossimo si 
riescano a rendere linkabili al database lessicografico i temini analizzati direttamente nel testo greco del papiro tanto in DCLP quanto in Papyri.info, in modo da ottenere una connessione sistematica tra questi e le schede lessicali.

Infine, dopo il nome dell'autore, il lemma termina con una lista cliccabile dei termini connessi al vocabolo principale (diminutivi, varianti, derivati, forme latine), che sono essi stessi ricercabili attraverso la lista alfabetica nella homepage.

È essenziale ribadire la stretta interconnessione con la banca dati testuale CPGM Online, al punto che Medicalia Online può essere considerato un supplemento e un ampliamento delle edizioni digitali dei papiri medici greci. Il tema dell'integrazione tra il database lessicografico e quello testuale porta inoltre a evidenziare la sostanziale rilevanza di una disamina lessicografica approfondita per un'altrettanto approfondita comprensione del testo. Uno studio 'verticale' della microlingua medica e della terminologia tecnica è in grado, infatti, di apportare contributi fondamentali per penetrare adeguatamente nei meandri del testo. Questo fa sì che la connessione tra i lemmi di Medicalia e le edizioni digitali dei papiri medici produca un mutuo arricchimento delle due banche dati, e metta in luce uno dei massimi vantaggi degli strumenti digitali: la possibilità di lavorare direttamente 'sul' testo e 'attraverso' il testo, così contribuendo a una più puntuale cognizione e interpretazione di esso. Questo aspetto potrebbe essere ulteriormente accresciuto grazie a un'indagine linguistica ancora più ampia, che coniughi l'analisi lessicale con quella della struttura morfosintattica del testo attraverso l'applicazione di differenti livelli di annotazione linguistica. L'annotazione ha infatti assunto un ruolo cardine nell'analisi linguistica di corpora testuali, permettendo di aggiungere informazioni di carattere (meta)linguistico alle diverse porzioni di un testo ${ }^{30}$. Considerando quindi come un corpus a tutti gli effetti la totalità dei papiri medici greci che ci sono pervenuti, l'applicazione ad esso dell'annotazione linguistica, svolta soprattutto mediante il treebank, può consentire di condurre un'analisi lessicale, fraseologica e sintattica estesa, in grado di permettere di risolvere problemi di interpretazione, e di pervenire a una più raffinata consapevolezza del significato del testo ${ }^{31}$. Tutto questo fa sì che il testo venga esplorato in modo immediato, dinamico e interattivo. Di fatto, immediatezza, dinamismo e interattività si annoverano tra le caratteristiche distintive delle edizioni digitali, nonché della stessa interconnessione tra la banca dati testuale e il database lessicografico di Medicalia. L'obiettivo, cui già si accennava, di creare una connessione sistematica dei vocaboli esaminati che compaiono nelle edizioni digitali e il lemma corrispondente in Medicalia contribuirebbe a rifinire la definizione di un termine

\footnotetext{
${ }^{30}$ Sull'annotazione linguistica cf. ad esempio Biber/Conrad/Reppen, Corpus Linguistics; Facchinetti, Description; Kuebler/Zinsmeister, Corpus Linguistics.

${ }^{31}$ Cf. Reggiani, Corpus, 2. Sull'uso dell'annotazione linguistica in settore antichistico e papirologico, cf. anche Reggiani, Digital Papyrology I, 180-6 con bibliografia nella n. 13.
} 
in un particolare papiro, quanto anche, non meno, a contestualizzarlo in tutte le fonti che lo attestano.

Il motivo dell'integrazione e dell'interconnessione tra il database principale e quello lessicografico aggiunge ulteriore evidenza al contributo di un minuzioso esame lessicale a una potenziata conoscenza testuale. Nella fattispecie, un approccio lessicografico 'verticale' può concorrere significativamente a rafforzare una duplice consapevolezza dell'oggetto lessicale in analisi rispetto al tessuto testuale in cui è inserito, ovvero 'concreta' da un lato e 'testualefilologica' da un altro $^{32}$. Infatti, come già messo in luce, la metodologia interdisciplinare e criticamente comparativa di Medicalia Online permette di indagare anche la dimensione più strettamente concreta che concerne il termine $\mathrm{e}$ il suo contesto. Un aspetto che rende quasi possibile 'visualizzare' la realtà materiale e 'pratica' che riposa sotto e al di là delle parole. A ciò contribuisce massimamente la seconda sezione del "Commentary" (C 2), che si focalizza esplicitamente sul lato concreto dell'oggetto lessicale. In tal modo, questa sorta di 'archeologia delle parole', mirante a 'rivitalizzare' il passato, rende gli stessi testi antichi più 'vivi' e i vocaboli che li compongono quasi 'tangibili'. Al contempo, un'analisi del termine condotta in tutte le sue attestazioni scritte dimostra un assai elevato potenziale nell'accrescere, come si accennava, la nostra consapevolezza dei testi antichi e delle questioni filologiche che li riguardano, e che si presentano talvolta alquanto problematiche. Ciò incrementa l'interpretazione tanto contestuale quanto filologica dei testi antichi di medicina, in misura tale da fornire lo spunto, talvolta, per nuove letture, nonché per individuare ed emendare possibili falle nelle edizioni ufficiali, come pure nella stessa tradizione manoscritta, nel caso degli scritti degli autori medici. L'esegesi critica di questi testi è infatti così strettamente dipendente dalla comprensione della terminologia medica che in essi appare, che il raggiungimento di una più esatta definizione dei vari elementi del vocabolario tecnico non può che consolidare la consapevolezza filologica del contesto testuale in cui i termini technici ricorrono. Vi è anche da sottolineare il fatto che la tipologia di studio elaborata per Medicalia Online consente di sviluppare un approccio all'oggetto lessicale esaminato che può essere definito tanto 'olistico' quanto 'atomico'. L'analisi è infatti condotta in modo 'olistico', nel senso che il termine è studiato meticolosamente in tutte le sue occorrenze, al fine di acquisire un quadro del significato o delle diverse sfumature semantiche che sia il più particolareggiato possibile. D'altro lato, un'ottica 'atomica' comporta di cogliere la parola nella sua verticalità, sviscerandone gli aspetti di dettaglio. Un tale approccio incoraggia, per così dire, un 'doppio movimento': dal testo alla parola - lo studio 'olistico' del termine in tutte le fonti scritte che lo attestano conduce a una più

\footnotetext{
${ }^{32}$ Per questi aspetti con alcuni case studies illustrativi, si rimanda a Bonati, Experience, 159-69.
} 
accurata comprensione del termine stesso - e dalla parola al testo e alla tradizione testuale. In questo modo, da una conoscenza 'verticale' del vocabolo deriva una altrettanto 'verticale' conoscenza in primis del testo medico su papiro che funge da base di partenza per la ricerca, ma anche, assai spesso, di alcuni (almeno) dei passi di autori medici che adoperano quel termine.

\section{Conclusioni}

L'esperienza di uno strumento per lo studio lessicale come Medicalia Online illustra il ventaglio delle prospettive e i differenti gradi di utilità di un database lessicografico connotato da un approccio metodologico di così ampio respiro. Per ricapitolare, si richiamino l'utilità e i risultati potenziali che un metodo di analisi comprensivo, dialogante e interdisciplinare può offrire ai diversi campi della ricerca antichistica: l'utilità insita nella natura di una risorsa digitale, che si traduce in dinamismo, flessibilità, possibilità di revisione, miglioramento e aggiornamento di quanto si è già scritto; l'utilità di scandagliare il mondo antico con uno sguardo aperto e ricettivo, che fa sì che uno strumento come Medicalia Online possa portare proficui contributi alla papirologia medica, quanto anche, contestualmente, agli altri settori 'in dialogo' con essa, per redarre i lemmi con un assetto 'verticale'; l'utilità di un layout che è al contempo innovativo e userfriendly nonostante il cospicuo quantitativo delle informazioni coinvolte; l'utilità, ovvero il mutuo arricchimento, che deriva dall'interconnessione tra le schede di Medicalia Online e le edizioni digitali dei testi medici su papiro, e che concorre significativamente a una più raffinata e profonda comprensione testuale, che si muove sul doppio binario di una consapevolezza intrinsecamente filologica, nonché concreta e materiale dell'oggetto lessicale indagato. Ciò risulta da un lato nello sforzo di ricostruire, o perlomeno delineare, la realtà fisica nascosta sotto le parole, e dall'altro incoraggia una più attenta esegesi dei testi medici attraverso una - altrettanto attenta - migliore conoscenza delle parole e dei loro valori semantici. Il fine ultimo si configura come un ponte sospeso tra il passato e il presente, le cui fondamenta sono gli apporti simultanei forniti dalle varie discipline, da cui si innalza lo scheletro di una conoscenza potenzialmente 'integrata' e 'inclusiva' del mondo antico, il quale viene colto come entità non 'morta', o - per quanto possibilie - 'viva', o almeno 'vivida', e ancora in grado di comunicare. 


\section{Bibliografia}

Biber/Conrad/Reppen, Corpus Linguistics = Douglas Biber / Susan Conrad / Randi Reppen, Corpus Linguistics, Investigating Language Structure and Use, Cambridge 1998.

Bonati, Composti = Isabella Bonati, Tra composti, suffissi e neologismi nella microlingua della medicina: alcuni specimina tratti dai papiri, in: N. Reggiani / F. Bertonazzi (edd.), Parlare la medicina: fra lingue e culture, nello spazio e nel tempo. Atti del Convegno Internazionale (Parma, 5-7 settembre 2016), Firenze 2018, 30-51.

Bonati, Dictionary = Isabella Bonati, Medicalia Online: An Electronic Dictionary of Technical Terms in Medical Papyri, in: Proceedings of the 28th International Congress of Papyrology (Barcelona, 1-6 August 2016), Barcelona, forthcoming.

Bonati, Experience = Isabella Bonati, Digital Papyrological Editions and the Experience of a Digital Lexicographical Database: The Case of Medicalia Online, in: N. Reggiani (ed.), Digital Papyrology II: New Methods for the Digital Edition of Ancient Greek Papyri, Berlin/Boston 2018, 149-73.

Bonati, Lessico = Isabella Bonati, Il lessico dei vasi e dei contenitori greci nei papiri. Specimina per un repertorio lessicale degli angionimi greci (APF Beihefte 37), Berlin/Boston 2016.

Bonati, Metafora = Isabella Bonati, L'uso della metafora nella microlingua greca della medicina, in: D. Astori (ed.), La metafora e la sua traduzione. Fra riflessioni teoriche e casi applicativi, Parma 2016, 84-100.

Bonati, Parola = Isabella Bonati, La parola delle cose. Nuove voci dal passato dei papiri, in: N. Reggiani / A. Bovo (edd.), Papiri, medicina antica e cultura materiale. Atti della Giornata in ricordo di Isabella Andorlini (Parma, 26 gennaio 2017), Parma 2018, 89-96.

Facchinetti, Description = Roberta Facchinetti, Theoretical Description and Practical Applications of Linguistic Corpora, Verona 2007.

Gentili, Poesia = Bruno Gentili, Poesia e pubblico nella Grecia antica. Da Omero al V secolo, Milano 2006 (ed. aggiornata).

Grmek, Maladies = Mirko D. Grmek, Les maladies à l'aube de la civilization occidentale, Paris 1983.

Hanson/Mattern, Catechism = Ann E. Hanson / Susan P. Mattern, Medical Catechism, in: I. Andorlini (ed.), Greek Medical Papyri I, Firenze 2001, 71-83.

Hübenthal, Thought = Ursula Hübenthal, Interdisciplinary Thought, "Issues in Integrative Studies" 12 (1994), 55-75.

Klein, Interdisciplinarity = Julie Klein, Interdisciplinarity: History, Theory, and Practice, Detroit 1994.

Klein, Mapping = Julie Klein, Mapping Interdisciplinary Studies, Washington 1999.

Klein/Newell, Studies = Julie Klein / William H. Newell, Advancing Interdisciplinry Studies, in: W. H. Newell (ed.), Interdisciplinarity: Essays from the Literature, New York, 3-22.

Kuebler/Zinsmeister, Corpus Linguistics $=$ Sandra Kuebler / Heike Zinsmeister, Corpus Linguistics and Linguistic Annotated Corpora, London/New York 2014.

Maravela, Micro-Language = Anastasia Maravela, Medical Micro-Language: The Contribution of Papyri to the Study of Medical Greek, in: N. Reggiani / F. Bertonazzi (edd.), Parlare la medicina: fra lingue e culture, nello spazio e nel tempo. Atti del Convegno Internazionale (Parma, 5-7 Settembre 2016), Firenze 2018, 12-29.

Milgram, Thinking = Stanley Milgram, Interdisciplinary Thinking and the Small World Problem, in: M. Sherif / C. W. Sherif (eds.), Interdisciplinary Relationships in the Social Sciences, Chicago 1969, 103-20.

Newell, Theory = William H. Newell, A Theory of Interdisciplinary Studies, "Issues in Integrative Studies" 19 (2001), 1-25.

Newell, State = William H. Newell, State of the Field: Interdisciplinary Theory, "Issues in Interdisciplinary Studies" 31 (2013), 22-43. 
Nissani, Cheers $=$ Moti Nissani, Ten Cheers for Interdisciplinarity: The Case for Interdisciplinary Knowledge and Research, "Social Science Journal" 34 (1997), 201-16.

Pagnotta, Lezione = Fausto Pagnotta, La lezione dei classici greci e latini nell'era globale, "Studium" 6 (2011), 847-55.

Reggiani, Corpus = Nicola Reggiani, The Corpus of Greek Medical Papyri and Digital Papyrology. New Perspectives from an Ongoing Project, in: M. Berti / F. Naether (edds.), Altertumswissenschaften in a Digital Age - Egyptology, Papyrology and Beyond. Proceedings of a Conference and Workshop in Leipzig (November 4-6, 2015), Leipzig 2016, URL: <http:// nbn-resolving.de/urn:nbn:de:bsz:15-qucosa-201726>.

Reggiani, Digital Papyrology I = Nicola Reggiani, Digital Papyrology I. Methods, Tools and Trends, Berlin/Boston 2017.

Repko, Research = Allen Repko, Interdisciplinary Research: Process and Theory, Thousand Oaks 20112.

Ruscio, Specializations $=$ Kenneth P. Ruscio, Bridging Specializations: Reflections from Biology and Political Sciences, "Review of Higher Education" 10 (1986), 29-45. 